\title{
Martin Schubert
}

\section{Johannes Rothe als didaktischer Chronist}

In der Renaissance der Beschäftigung mit Johannes Rothe, die in den letzten Jahren zu erleben ist, wird besonders die Editionslage ganz neu aufgestellt. Mit den Editionen des >Elisabethlebens<, der >Eisenacher Chronik ,Landeschronik< liegen drei Werke erstmals in wissenschaftlichen Ausgaben

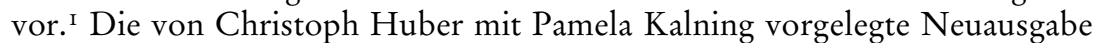
des >Ritterspiegels< erschließt das Werk und Rothes Arbeitsweise eingehend. ${ }^{2}$ Bald ist mit dem Abschluss der Ausgabe der >Geistlichen Brustspange < durch Jens Haustein zu rechnen, ${ }^{3}$ die endlich einen Text veröffentlicht, der schon vor einem Dreivierteljahrhundert in den Druck gebracht werden sollte, ${ }^{4}$ und damit die letzte Lücke im Zugang zu Rothes Werken schließt. Es besteht also jetzt die Gelegenheit, das Oeuvre Revue passieren zu lassen und grundlegende Fragen, wie die nach seinem Einsatz didaktischer Sprechweisen, in Querschnitten zu untersuchen.

Zuvor sei der Hinweis erlaubt, dass auch die Grundlagenforschung noch offene Fragen bietet. Da Rothe Stadtschreiber, Kleriker und Schulmeister war, sind wir über ihn als Person gut unterrichtet und können seine Lebensstationen von rund I360 bis I434 nachvollziehen. Auch die Werke sind durch Indizienketten in eine akzeptable Reihenfolge gebracht worden, in der zum Beispiel die Rechtsbücher zur Stadtschreibertätigkeit geordnet werden (um I 384-I 397).5 Aufgrund von Bezügen können undatierte Werke an datierte angekoppelt werden; so wird das Elisabethleben in das letzte Lebens-

I Johannes Rothes Elisabethleben. Aufgrund des Nachlasses von Helmut Lomnitzer hg. von Martin J. Schubert und Annegret Haase, Berlin 2005 (Deutsche Texte des Mittelalters 85); Johannes Rothe, Thüringische Landeschronik und Eisenacher Chronik, hg. von Sylvia Weigelt, Berlin 2007 (Deutsche Texte des Mittelalters 87).

2 Johannes Rothe, Der Ritterspiegel. Herausgegeben, übersetzt und kommentiert von Christoph Huber und Pamela Kalning, Berlin, New York 2009.

3 Vgl. http://www2.uni-jena.de/philosophie/germlit/mediaev/dfgpro2.htm (die Projekthomepage).

4 Vgl. Ludwig Ahmling, Liber devotae animae. Ein neues Werk Johannes Rothes. Vorstudien zu einer Ausgabe des Gedichts, Diss. Hamburg 1933.

5 Siehe z. B. Pamela Kalning, Kriegslehren in deutschsprachigen Texten um I 400. Seffner, Rothe, Wittenwiler, Münster 2006 (Studien und Texte zum Mittelalter und zur frühen Neuzeit 9), S. 88. 
jahrzehnt gerechnet. Die drei Chroniken wiederum wurden anhand von Detailuntersuchungen zu Entlehnungsverfahren in die Reihe Eisenacher Chronik - Landeschronik - Weltchronik gebracht, welche durch den Abschluss der lateinischen >Historia Eccardiana< (wohl I4I4) als wesentlicher Vorlage einerseits, durch I42 I als Datierung der Weltchronik im Akrostichon andererseits eingegrenzt wird und über die Dedikation der Landeschronik eine weitere Stütze erhält. ${ }^{6}$ Die Eisenacher Chronik wird dabei üblicherweise vor der Landeschronik, also zwischen I4I4 und I4 I 8, eingeordnet. Die Schwierigkeiten der Einteilung zeigt ein unscheinbares Indiz: In der Eisenacher Chronik finden sich drei Verweise auf die sächsisch-wittenbergische Herzogswürde Markgraf Friedrichs IV. des Streitbaren von Meißen, die dieser erst 1423 erlangte, 7 ein Umstand, der im Rahmen der geläufigen Datierungen nur als „wohl spätere Interpolation“ 8 verstanden werden kann. Zwar ist eine Chronik gerade am Ende offen für Fortschreibungen und Aktualisierungen doch wäre es für unser Textverständnis fatal, hier am Werkende zu großzügig zu athetieren, da selbst der Eintrag zu dem Ratsherrn Reinhard Pinkernagel, der für die angenommene Rezipientenausrichtung grundlegend ist, erst wenige Zeilen nach den Verweisen auf die Herzogswürde steht. ${ }^{9}$ Kann es sich bei den bewussten Stellen um einen dreifachen, penetranten Fall von Interpolation handeln? Betrachten wir dazu das Umfeld der ersten Stelle, die Nennung der drei Söhne Friedrichs des Strengen:

Der eldister Friderich der wart eyn herczoge zcu Sachsin. Dy andern zcwene Wilhelm und Jorge dy storbin ane wibe und ane libis erbin. ${ }^{\circ}$

Neben der Herzogswürde wird also auch noch auf die Kinderlosigkeit der anderen beiden Brüder verwiesen, die erst bei ihrem Tod feststeht - bei Georg I 402 und bei Wilhelm I425. Zwei Bemerkungen weisen hier also, von der angenommenen Entstehungszeit aus gesehen, weit in die Zukunft. Wollte man konsequent sein, könnten nur beide Sätze gemeinsam als spätere

6 Grundlegend zu den Entlehnungen Hermann Helmbold, Johannes Rothe und die Eisenacher Chroniken des I 5. Jahrhunderts, in: Zeitschrift des Vereins für thüringische Geschichte und Altertumskunde (N. F.) 2I (I9I3), S. 393-452; vgl. Weigelt (Anm. I), S. LXXVI.

7 Weigelt (Anm. I), I 3 I.2 I-23, I 32.1 4 und I 32.27 f.

8 Weigelt (Anm. I), S. LXXVI, Anm. 248.

9 Weigelt (Anm. I), I 32.30-34; siehe dazu S. LXXVII mit Lit.: Auf diese Stelle stützen sich Matthias Werner, „Ich bin ein Durenc”. Vom Umgang mit der eigenen Geschichte im mittelalterlichen Thüringen, in: Identität und Geschichte, hg. von Matthias Werner, Weimar 1997, S. 79-104, hier S. 97; Volker Honemann, Johannes Rothe in Eisenach. Literarisches Schaffen und Lebenswelt eines Autors um I400, in: Autorentypen, hg. von Walter Haug und Burghart Wachinger, Tübingen 1991 (Fortuna Vitrea 6), S. 6988, hier S. 72; Hans Neumann, Rothe, Johannes, in: ${ }^{1}$ VL, Bd. 5, 1955, Sp. 995-1006, hier Sp. 998; Helmbold (Anm. 6), S. 443.

Io Weigelt (Anm. I), I 3 I.2 I-23. 
Interpolation angesehen werden, auch wenn sie ansonsten stilistisch unverdächtig sind. Genauen Aufschluss könnte nur eine möglichst komplette Diskussion aller Episoden des Chronikschlusses geben, die am besten auch die Überlieferungsvarianz der lateinischen Vorlage einbeziehen würde. ${ }^{\text {II }}$ Dabei sollte die Möglichkeit im Auge behalten werden, dass Rothe auch selbst bis nach $1425 \mathrm{zu}$ seiner Chronik beitragen und durch das Zufügen von Autorvarianten den Befund erschweren konnte. ${ }^{\mathrm{I} 2}$ Dies würde allerdings entweder die angenommenen Datierungen tangieren oder die bislang immer mitgeführte Vorannahme, dass Rothe jeweils ein umfangreiches Werk zu Ende führte, bevor er ein neues begann.

Nun könnte gefragt werden, warum aus größerem zeitlichem Abstand die Chronik nicht sogleich weiter fortgeschrieben wurde, näher bis an die Gegenwart des Chronisten. Hierzu ist ein Blick auf die jeweiligen Enden hilfreich, die teils durch spätere Zusätze verschleiert werden. Die Landeschronik reicht in der von Sylvia Weigelt erschlossenen Urform nur bis zum Tode des Landgrafen Balthasar I406; ${ }^{13}$ die Eisenacher Chronik gibt nach diesem Ereignis nur noch wenige Einzelheiten bis zur Datierung I409. ${ }^{14}$ Die Weltchronik enthält im überlieferten Text zwar Ereignisse bis I440, wobei es sich ja schon angesichts von Rothes Sterbedatum zum Teil um fremde Nachträge handeln muss; zudem ist der Text im Akrostichon auf I 42 I datiert. Die eindeutig durch das Akrostichon gebundenen Passagen allerdings reichen nur bis zum Kapitel 758, ${ }^{15}$ also bis zur Hochzeit der Anna von Schwarzburg mit

I Ein sonderbares Indiz ist der Umstand, dass die ansonsten parallel aufgebaute Passage in der Eisenacher Chronik I31.29-36, Landeschronik 88.21-32 und Weltchronik Kap. 73 of. (mit den drei Episoden Tod Friedrichs des Strengen, Tod seines Bruders Ludwig von Magdeburg und Landgraf Balthasar vor Brandenfels) nur in der Landeschronik 88.23-26 durch die Hochzeit Balthasars mit Margarethe unterbrochen wird deutlich als sekundärer Einschub markiert, da das aufgreifende syn bruder in $88.27 \mathrm{im}$ vorliegenden Text eher fälschlich auf Balthasar, seinen Schwiegervater, seinen Sohn oder seinen Schwiegersohn bezogen werden müsste, die alle zwischendurch genannt sind -, was allerdings in den Quellenverweisen bei Weigelt im gleichen Kapitel der 'Historia Eccardiana< nachgewiesen ist und also höchstens durch erneuten Rückgriff auf die Quelle als Einschub zu verstehen sein könnte.

I2 Man vergleiche mit diesem Hintergedanken die Angaben bei Weigelt (Anm. I), S. LXI-LXIV zu den Fassungen der Landeschronik, die mit zweifacher Fortsetzung bis ${ }_{4} 427$ und mit Kontaminationen ebenfalls Indizien für eine eng verschränkte Entstehungsgeschichte bieten.

I3 Weigelt (Anm. I), S. LXV: „Dieses Ende scheint mit Blick darauf gewählt, daß Balthasar der letzte thüringische Landgraf war, der auf der Wartburg residierte und dessen Tod einen gravierenden Einschnitt der herschafft von Doringen (6.198) markierte, auf die Rothes Chronik so explizit ausgerichtet war."

I4 Weigelt (Anm. I), I34.32-I 35.37 .

Is Kapitelangaben hier und im Folgenden immer nach der Ausgabe: Düringische Chronik des Johann Rothe, hg. von Rochus v. Liliencron, Jena I859 (Thüringische Geschichtsquellen 3), auch wenn deren Kapitelfolge mittlerweile als sekundär erwiesen 
Landgraf Friedrich dem Friedfertigen im Jahr I 407. Das bedeutet also - dies wurde bislang noch nicht genügend betont - dass alle drei Chroniken in ihrer ursprünglichen Form kaum über die Jahre I 406 / I 407 hinausreichten. Die Übernahme der Landgrafschaft durch Friedrich den Friedfertigen im Jahr I 406, der bis 1440 regierte, scheint also der Kulminationspunkt der Chroniken gewesen zu sein: als Übergang zur Herrschaft des zur jeweiligen Abfassungszeit aktuellen Herrschers, gewissermaßen als Übergang zur Zeitgeschichte. Dieser Teil der Geschichte wird nicht zum Kern der Chronik gerechnet, sondern erst in den unterschiedlichen Anhängen ausgeführt. Dabei bleibt die Option offen, dass diese Anhänge zum Teil oder gar großteils auf Rothe selbst zurückgehen können. Die bisher aufgestellten Thesen zu seiner Arbeitsweise, bei der verschiedene Werke auch parallel und mehrfach in Überarbeitungen über seinen Schreibtisch gehen konnten, ${ }^{16}$ lassen eine weniger lineare Entstehungsgeschichte möglich scheinen. Den Vorsatz zu nachträglichen Eingriffen in ein abgeschlossenes Werk, sofern seine Lebensdauer es zulässt, hat Rothe selbst im Versprolog zur Weltchronik formuliert. Dort erklärt er über die Landgräfin:

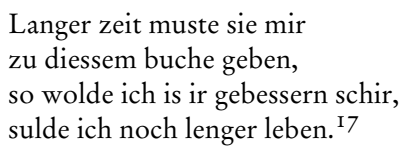

Rothes Bezugnahme zum Publikum und seine didaktische Vorgehensweise ist bereits mehrfach behandelt worden. Peter Strohschneider hat versucht, die Chroniken und das >Elisabethleben $<$ durch eine sozialhistorisch orientierte Lektüre den möglichen Rezipientengruppen zuzuordnen. ${ }^{18}$ Auf die angesichts von Rothes sehr sachorientiertem Stil notwendig hypothetischen An-

ist; vgl. Anm. 22. Bech versuchte, das Akrostichon auch in Kap. 759-773 wiederzufinden; seine Vermutungen konnten durch die neue Handschrift nicht bestätigt werden, siehe Pfeil (Anm. 22), S. 219 , Anm. 36.

I6 Vgl. zum Beispiel der Fassungen des >Elisabethlebens< Schubert/Haase (Anm. I), S. LIIIf.; zur eventuell parallelen Entstehung von Chroniken und >Ritterspiegel Kalning (Anm. 5), S. 89.

17 Liliencron (Anm. I 5), S. 6.

I8 Peter Strohschneider, Johannes Rothes Verslegende über Elisabeth von Thüringen und seine Chroniken. Materialien zum Funktionsspektrum legendarischen und historiographischen Erzählens im späten Mittelalter, in: Internationales Archiv für Sozialgeschichte der deutschen Literatur 23 (1998), S. I-29. 
teile solcher Folgerungen hat Christoph Huber hingewiesen. ${ }^{19}$ Zur Didaktik Rothes hat Henrike Lähnemann einen Beitrag zum >Ritterspiegel< vorgelegt, in dem sie die Führung der Rezipienten in das Werk hinein, die allegorische Matrix des Spiegels und die Organisation durch formale Großgliederung und assoziative Binnengliederung bespricht. ${ }^{20}$ Die formale Großgliederung macht sie am Akrostichon fest, das als begleitender Paratext ein für die Wendung zum Publikum prädestinierter Ort ist. Dass ein Akrostichon darüber hinaus noch Beziehungen zur didaktischen Wendung aufweist, soll im Folgenden unter einem unerwarteten Aspekt beleuchtet werden, und zwar innerhalb seiner Weltchronik. Chroniken unterrichten zwar über den Weltlauf und können dem Rezipienten Rückschlüsse und Erkenntnisse nahe legen, aber sie zählen mit Recht nicht zur didaktischen Literatur und bieten meist recht wenig explizite Unterweisung. Bei Rothe, der ja generell oft des Eklektizismus verdächtigt wird, wurde bereits mehrfach erwähnt, dass didaktische Redeweisen auch in die Chroniken einfließen. ${ }^{21}$ Diese sollen hier einmal im Detail beobachtet werden.

Aber zunächst zurück zu dem Akrostichon der Weltchronik. ${ }^{22}$ Die 758 Buchstaben des umfangreich und umständlich erläuternden Beitextes erstrecken sich über ebenso viele Kapitel. ${ }^{23}$ An jedem Kapitelanfang wird also ein

I9 Christoph Huber, Die Ritterweihe Landgraf Ludwigs IV. bei Johannes Rothe. Historiographischer Textbaustein und poetologische Aspekte, in: Literatur und Macht im mittelalterlichen Thüringen, hg. von Ernst Hellgardt, Stephan Müller und Peter Strohschneider, Köln, Weimar, Wien 2002, S. 165-177, hier S. 174.

20 Henrike Lähnemann, Didaktische Verfahrensweisen in Johannes Rothes ,Ritterspiegel', in: Hellgardt, Müller, Strohschneider (Anm. 19), S. I79-190, hier S. I 80. Vgl. weiter zur Didaktik das Kapitel „Wertorientierte Adelsdidaxe in Johannes Rothes ,Ritterspiegel'“ bei Kalning (Anm. 5), S. 87-I 39.

2I Siehe Huber (Anm. 19), S. I72: „moralische Belehrung und geistliche Erbauung fließen quer durch Rothes Chroniken und Legenden ineinander"; Weigelt (Anm. I), S. XXI: „Über Kommentare versucht der Chronist zudem, das Handeln und Verhalten seiner Protagonisten zu erklären oder auch moralisch-didaktische Unterweisung zu erteilen.“

22 Das Akrostichon wurde von Fedor Bech in der Rezension zu Liliencron (Anm. I 5 ), in: Germania 5 (1860), S. 226-247, rekonstruiert; wiederabgedruckt bei Lähnemann (Anm. 20), S. I85f. Die von Brigitte Pfeil entdeckte Wernigeröder Handschrift bestätigt aufs Feinste Bechs Rekonstruktion; siehe Brigitte Pfeil, Die ,Thüringische Weltchronik' des Johannes Rothe aus der Büchersammlung der Fürsten zu StolbergWernigerode (Halle, ULB, Stolb.-Wernig. Zb 32), in: Mittelalterliche Sprache und Literatur in Eisenach und Erfurt. Beiträge der Erfurter Tagung am 23. 8. 2006 anlässlich des 70. Geburtstags von Rudolf Bentzinger, hg. von Martin J. Schubert, Annegret Haase und Jürgen Wolf (Kultur, Wissenschaft, Literatur. Beiträge zur Mittelalterforschung), Frankfurt a. M. u. a. 2008, S. 207-229.

23 Wohl aufgrund von Textschäden, die auch nicht durch die neue Handschrift behebbar sind, ist nicht recht verständlich, wie die Vollendung des Textes samt des Akrostichons vorzustellen ist. Sicherlich gilt nicht „Das Werk wurde also am 2I. Juni 142 I vollendet“ (Lähnemann [Anm. 20], S. I86, Anm. I3), denn das würde bedeuten, dass sämtli- 
zuvor festliegender Buchstabe benötigt, was eine ungewöhnliche Kapiteleinleitung erfordert. Während die meisten Abschnitte der Eisenacher Chronik und der Landeschronik mit Do man schreib oder mit In dem selben jare sowie analogen Ausdrücken beginnen, ist in der Weltchronik beispielsweise in Kap. 739 ein $H$ erforderlich. Nur hier beginnt der Bericht zum Tod Adolfs von Mainz mit Heiligenstat beweynete seynes herren bischouff Adolffus von Mentze ende, ${ }^{24}$ wodurch ein außergewöhnlicher Aspekt, nämlich eine emotional gefärbte Betonung des Kummers der Einwohnerschaft angesichts des Sterbefalls, in den Text eingebracht wird. Ähnlich ausgefallen sind solche Einleitungen:

Kap. 685 Rache ted unsser herre Jhesus Cristus obir seyne viende alsso man zalte noch Cristus gebort I 348 jar ...

Kap. 700 Trost unde hulffe suchten die von Kyndelbrucken an den lantgraven zu Doryngen

Kap. 7I 2 Trost unde hulffe suchte der babiste an keisser Karlen

Kap. 728 Totliches obil geschach ...

Kap. 735 Redeliche sache gewan bischouf Adolff von Mentze ...

Bereits die wenigen Beispiele - die natürlich geborgen sind zwischen zahlreichen usuellen Einleitungen wie Es geschach oder Noch Cristus gebort, sofern die entsprechenden Buchstaben gefordert sind - zeigen einen Rekurs in formelhafte Rede (Trost unde bulffe), die aber gerade nicht zu den dem Genre sonst üblichen Formeln zählt. Vielmehr treten hier Bewertungen und emotionale Färbungen hinzu; ein Vorgang, der nur auffällt, wenn man sich vor Augen ruft, dass Rothe in der chronistischen Wiedergabe sonst kaum jemals Betroffenheiten vermeldet oder eine Situation mit einer Bewertung versieht. Nur bei den allergröbsten Bestialitäten ist ihm eine Stellungnahme zu entlocken; so kommentiert er bei der Erzählung von der Belagerung Jerusalems (Kap. 84) den Umstand, dass zwei Frauen ihre eigenen Kinder gekocht und verspeist hätten: Es ist erbermiglich, das man is sprechen ssal.

Der Kapitelanfang hebt sich mehrfach von dieser Reserviertheit ab. Die genannten besonderen Stellungnahmen korrespondieren mit einer besonderen Behandlung der jeweils ersten Sätze. Es ist hier vielleicht, analog zum Reimzwang, von einem Akrostichon-Zwang zu sprechen, der jeweils am Kapitelanfang eine kleine Verschiebung verursacht, die dem Autor Anlass bietet, aus seinem gewohnten Duktus auszubrechen und einen Registerwechsel vorzunehmen. Diese Verschiebung scheint auch für didaktische Redeweisen wesentlich zu sein.

In den Chroniken sind insgesamt didaktische Einflechtungen, Bezugnahmen auf das Publikum oder auf den Chronisten sehr selten. Da Rothe be-

che Kapitel ab den zur Tagesdatierung gehörigen Akrostichonbuchstaben an diesem Tag verfasst sein müssten, immerhin Kapitel 656-758.

24 Vgl. die konventionellen Einleitungen der parallelen Stellen der anderen Chroniken: Eisenacher Chronik I32, I7f.; Landeschronik 89,7f. 
kanntlich in seinen Chroniken auch dort, wo er Ereignisse selbst erlebt hat, als Person ganz hinter den chronistischen Rahmen zurücktritt, ${ }^{25}$ ist jeder Bezug auf den Autor ein nennenswertes Indiz. Durchsucht man zunächst das Textinnere der Abschnitte der Weltchronik, so findet sich dort wenig Bemerkenswertes. Selbstnennungen treten eigentlich nur auf, wo eine Wendung gegen alle Schriftquellen zusätzliche persönliche Autorität zu erfordern scheint, so wenn Rothe versucht zu erläutern, dass die Kinder Noahs auch bis Thüringen gekommen sein könnten:

das ich meyne das sie gar vil lant durchwandert han unde villeichte yn dem lande zu Doringen ouch ir eyn teil gewest synt, wenn ich vornomen habe, das man bey Salza vor gezeiten yn eyme steine funden hat eynen eichen zunstecken, der noch der syntflut alsso tief yn der erden noch yn dem steyne sich nicht behalten mochte. (Kap. I 5 )

An dieser Stelle nennt sich der Chronist gleich zweimal: um seine Meinung zur vorsintflutlichen Besiedlung Thüringens kundzutun und um seine eigene - wenn auch indirekte - Zeugenschaft für den im Grund und Gestein gefundenen Zaunpfahl anzubringen. Neben der Deutung dieser Selbstnennung als Methode der Authentifizierung ließe sich auch annehmen, dass sie - nicht notwendig absichtlich - beim Aussetzen schriftlicher Quellen den Untergrund eigentlich dünner Gewährleistung markiert. Auf einen solchen $\mathrm{Zu}$ sammenhang könnte eine explizite Unwissenheitserklärung deuten, die bei einer weiteren Selbstnennung zu finden ist. Die Unklarheit bezüglich der Könige, die dem antiken Trier untertänig waren, verantwortet der Chronist selbst (Kap. 28): wie sie aber bey namen gebeissen waren unde was slos ader stete sie buweten, des habe ich nicht vor warheit erfaren.

Rar sind ebenfalls Bemerkungen zur Redeorganisation; sie beschränken sich auf Erklärungen, dass einige Details nicht im Einzelnen ausgeführt werden:

Kap. 33 ... ander zyrunge, die nicht alle uss zu sagen stehen von ir meynige unde gute

Kap. 45 Sulde man von den andern kirchen schreiben [...], das hynderte hie zu ssere.

Kap. 679 Von ir togunt [...] were wol vaste zu sagen, die sie begangin hat, das hie alles zu langk worde uss zu richten.

Es ist kein Zufall, dass die genannten Stellen alle in letzten Sätzen von Kapiteln stehen: Solche Verweise sind ein probates Mittel, Abschweifungen einzukürzen, wobei die Formulierung, etwas sei nicht us zu sagen, also nicht bis zu Ende zu erzählen, bei Rothe schon topischen Charakter hat. ${ }^{26}$ Auch sehr

25 Siehe Volker Honemann, Johannes Rothe und seine >Thüringische Weltchronik«, in: Geschichtsschreibung und Geschichtsbewußtsein im späten Mittelalter, hg. von Hans Patze, Sigmaringen 1987 (Vorträge und Forschungen 31), S. 497-522, hier S. 519f.; vgl. Lähnemann (Anm. 20), S. г 86.

26 Man vergleiche hierzu die analogen Belege Eisenacher Chronik i I0.34f.; Landeschronik 54.10; ; Elisabethleben< V. 875, 1728, $288 \mathrm{I}$. 
selten ist die Publikumsanrede, die ebenfalls der Redeorganisation dienen kann (Kap. I4a): Nu musse wir furt die sippe von Adam rechen bis uff Noen.

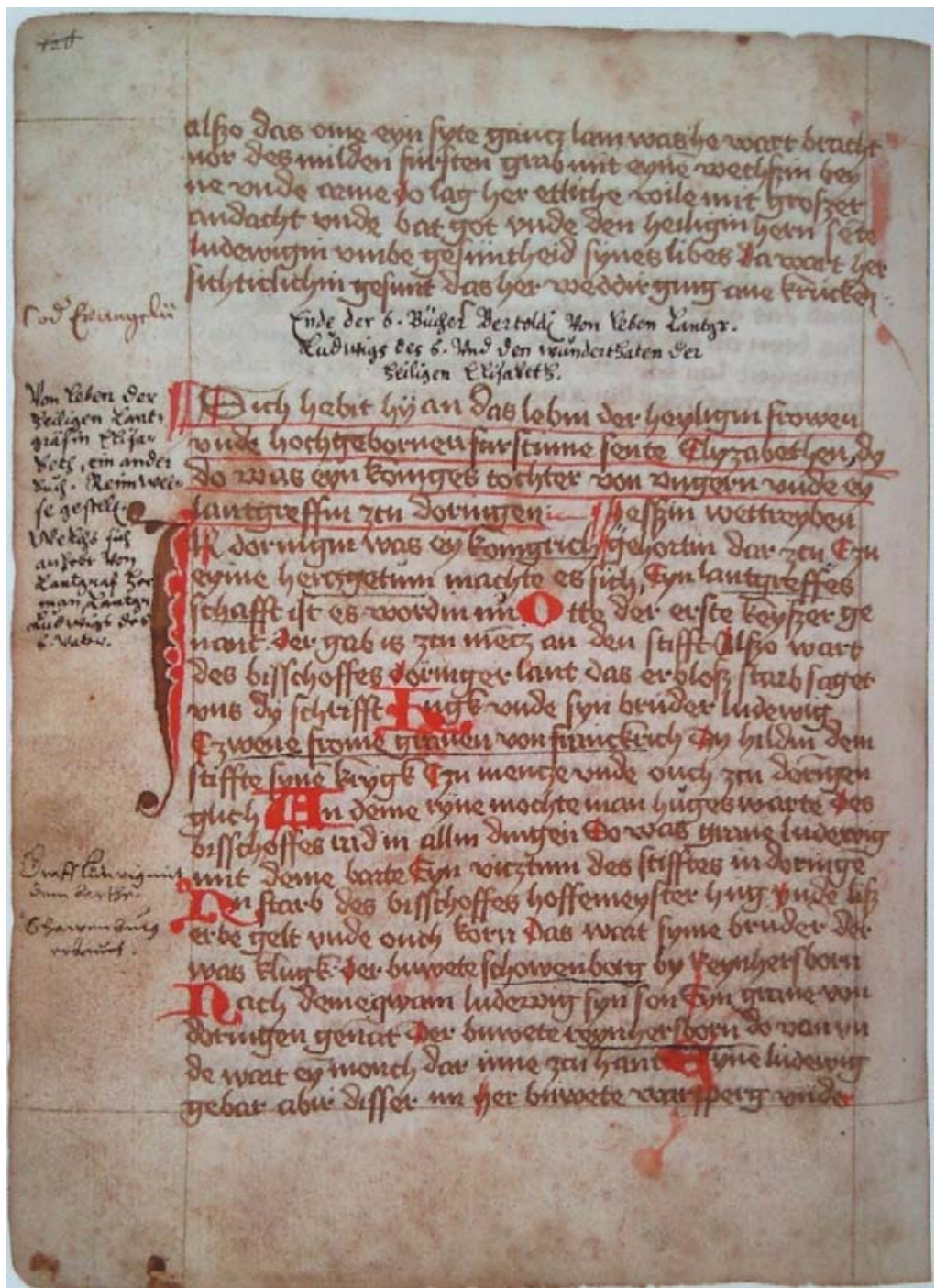

Akrostichongebrauch bei Johannes Rothe. Erste Seite des `Elisabethlebens` (IOHANNE[S SCOLAST]) aus Erfurt/Gotha, Universitäts- und Forschungsbibliothek - Forschungsbibliothek Gotha, Chart. B 52, f. 63v. Vgl. die Edition von Haase / Schubert 2005 (Anm. I), Tafel 2. 
Es bietet sich ein anderes Bild, wenn man diese Phänomene in den ersten Sätzen der Abschnitte verfolgt; ${ }^{27}$ hier kommen sie deutlich häufiger vor. Eine Anzahl von redeorganisierenden Stellen liegt vor, worunter allerdings wieder die Selbstnennungen marginal bleiben (Kap. I4): Von Adame muss ich aber vorder sagen. Eine explizite Aussage zum Fortgang der Darstellung steht am Anfang von Kap. 24: Genugk ist gesagit yn den landen yensseit meris von den geborten, wir sullen ouch vorder komen zu der herschaft bie dissit des meris yn dutzschen landen. Apart ist dieser Beleg, weil das Versprechen, nun zu den deutschen Herrschaftsgeschlechtern vorzustoßen, im angebrochenen Kapitel (und in den beiden folgenden) noch gar nicht eingelöst wird, sondern erst im Kapitel 27. Abgesehen von dem treffenden einleitenden Buchstaben wirkt die Bemerkung hier leicht deplaziert.

Auch in den ersten Sätzen von Kapiteln ist der erklärte Verzicht auf Details anzutreffen, wie er oben mehrfach zum Kapitelschluss zu sehen war (Kap. 43): Alle tempel zu Rome zu beschreiben [...], der mer wen tussent synt, das were besundern alzu vordrossen unde arbeitsam. Ebenso liegt der genau entgegengesetzte Fall vor, dass nämlich gerade die Notwendigkeit eines detaillierteren Berichts betont wird (Kap. 45): Nicht toug aller dynge zu sweigen von den kirchen zu Rome... In der Zusammenschau der beiden Anfänge wird der parallele Aufbau deutlich: Von den heidnischen Tempeln kann nicht vollständig berichtet werden, von den christlichen Kirchen sollte nicht geschwiegen werden. Häufiger als innerhalb der Kapitel treten redeorganisierende Publikumsanreden auf:

Kap. I 2 Horet vort von Lameche

Kap. $27 \mathrm{Nu}$ sullen wir vort horen, wie es qweme umbe Trebetan ...

Kap. 28 Prufet nu unde mercket, wie sich die dutzsche sprache unde die herschaft hat erhabin yn dissen landen ...

Kap. $49 \mathrm{Nu}$ sullen wir vort sagen wie die Romer yre stat bilden ...

Kap. 83 Horet nu ouch von den Juden ...

Die bislang angeführten Beispiele lassen sich nicht ohne weiteres als Hinweise auf ein didaktisches Anliegen vereinnahmen. Aber gerade am Kapitelanfang in dieser Position finden sich dann auch Sentenzen, die ja ebenso in den Versprologen und in den didaktischen Werken wie dem >Ritterspiegel eingesetzt werden:

Kap. 29 Rache geschiet gerne obir die hochfertigen unde obirmutigen

Kap. 60 Es hat die geierheit von naturen keyne genuge.

Kap. 7I Hie yn diessen landen heldit man das vorwunder, das ynn andern landen nymanden wunderlichen hat.

27 Die folgenden beispielhaften Belege sind vollständig für 200 Kapitel, also ein gutes Viertel des Gesamttextes. Ausgewählt sind dafür die Kap. I bis 100 und $658-758$, also Abschnitte vom Anfang und vom Ende des durch das Akrostichon gesicherten Bestands. 
Kap. 742 Truwe unde warheit vynden die fursten itzunt selden an yren mannen, alsso sie etzwanne vor jaren gethan habin.

Der vollständigste Ausflug in didaktische Redeweisen ist die Einleitung des Kapitels 736 der Weltchronik, dem Bericht über die fatale Neuorganisation des Eisenacher Rates. Das Akrostichon erfordert hier ein $O$ :

Obir alle dyngk ist gut unde nutze den steten eyntrechtigkeit unde gehorssam, wen von dissen zwen werden cleyne stete mechtigk unde reiche unde von zweitracht unde ungehorssam vorterben grosse stete unde vor armen.

Hier und im anschließenden Kapitel findet sich in konzentrierter Form die Palette der aus den übrigen Werken bekannten didaktischen Redeweisen. Hier steht der verallgemeinernde wertende Merkspruch (... ist gut unde nutze ...) ebenso wie die dichotomische Ableitung allgemeiner Folgerungen (... werden cleyne stete mechtigk ... vorterben grosse stete). Assoziativ angeknüpft wird an den Begriff der zweitracht, diese führe dazu, dass die Gehässigen andere Leute schädigen wollen. Eingeschoben wird die in Rothes belehrenden Texten häufige ${ }^{28}$ Autoritätenberufung: Dis schreibet Aristotilis yn seynen buchern von den steten. ${ }^{29}$ Danach wird eine weitere assoziative Verallgemeinerung zugefügt: Nach Ämtern streben nur, die andere bedrängen oder schädigen wollen oder für sich selbst Gewinn oder Prestige erwarten. Erst auf dieser Grundlage, einer negativen Staatenlehre, wird die eigentliche Erzählung entfaltet. Der Rezipient folgt dem Chronisten Schritt für Schritt in eine absurde Regierungsform und ist damit vorbelastet für die anschließende Erzählung der Eisenacher Bürger, die sich durch ein Geschenk an den Landgrafen Balthasar einen Ratssitz erschlichen hatten. Die daraus resultierende Handlungsunfähigkeit des Rates wird auf der Grundlage des moralisch-didaktischen Vorlaufs als Niedergang der gesamten Tugenden des Gemeinwesens dargestellt: alsso vorgyngk dor von der gehorssam eyntrechtigkeit gesetze ynnunge unde allis das sich zu redelichkeit gezubet. Hier äußert sich mit aller ihm möglichen Entrüstung Rothe mit dem Ethos seiner Erfahrung als Stadtschreiber. Die differenzierte Ausrichtung seines Textes auf ein intendiertes Publikum bleibt aber in jedem Fall gewahrt: Die Katastrophe wird nicht geschildert, ohne dass der Landgraf Balthasar entlastet wird. Immerhin war er der Schwiegervater der Landgräfin Anna von Schwarzburg, der die Weltchronik gewidmet ist; von ihm heißt es: Der gutliche herre geborchte yren worten unde satzte sie yn den rad unde machte sie gewaldigk, der sete unde gelegenbeit her nicht enkante. Die Gutgläubig-

28 Vgl. Lähnemann (Anm. 20), S. I 88.

29 Vgl. die analogen Berufungen auf Aristoteles im >Ritterspiegel (Register in der Ausgabe [Anm. 2], S. 450 und Kommentar S. 279), in der Landeschronik 4.I 33; in der >Geistlichen Brustspange V. 3082, 3432, 4I 84, 4530, 52 I 2. Ich danke Jens Haustein, dass ich den Text seiner Ausgabe (Anm. 3) schon vorab einsehen konnte. Vgl. zu Berufungen auf Aristoteles Lähnemann (Anm. 20), S. I 88. 
keit des gutlichen herren gegenüber den bösen Bürgern, über deren Eigenschaften er sich nicht ihm Klaren war, scheint hier das Einzige zu sein, was dem vorherigen Fürsten angekreidet werden könnte. ${ }^{30}$

Doch dies ist nur eine kleine, höfliche Konzession. Rothe wird an dieser Stelle, wo der Rat als eine ihm zentrale Ordnungsinstitution bedroht war, grundsätzlich und entfaltet, zum einzigen Mal in der Weltchronik, das Spektrum seiner didaktischen Verfahren. Ein wesentliches Thema erfordert ein entschiedenes Vorgehen, und als Anstoß und Ausgangspunkt mag ein einziger Akrostichonbuchstabe genügen.

Rothe verfasst Werke in verschiedenen Gattungen, wobei gelegentlich Verfahren und Redeweisen eingemengt werden, die in einer anderen Gattung üblich sind. Ähnlich wie es im Elisabethleben zur Kreuzung von legendarischen und chronistischen Elementen kommt, ${ }^{3 \mathrm{I}}$ so greifen auch didaktische Verfahren in jene seiner Werke ein, die gattungsmäßig nicht zur didaktischen Literatur zählen. In den Chroniken ist dies im Allgemeinen sehr selten, aber es gibt einen Bereich, in dem sich ein freies Forterzählen gegenüber dem strengen chronistischen Fortgang einschleicht. Dieser Bereich freier Gestaltung entsteht auffälligerweise gerade in den Partien der formalen Bindung mit ihren Notwendigkeiten: in den Sätzen, die am Akrostichon hängen, und in den Reimprologen. Hier treten Sentenzen und Autoritätenberufungen auf; gerade in diesen einzigen assoziativ freien Passagen der Chronik wird Rothe grundsätzlich. Hier scheint immer wieder das hindurch, was Rothes Wendung zu seinem Publikum ganz wesentlich bestimmt ${ }^{32}$ und was er ungeachtet seiner verschiedenen Publikumsgruppen immer wieder verwendet: seine didaktische Ader, die mit einem festen Satz literarischer Mittel die Belehrung und Erbauung in alle Gattungen einmischt.

30 Vgl. Weigelt (Anm. I), S. XII zu den Harmonisierungsbestrebungen Rothes, die Weigelt dafür verantwortlich macht, dass die Episode in der >Eisenacher Chronik ‘ völlig unterschlagen wird. In den Ratsgedichten, die sich auf das gleiche Ereignis zu beziehen scheinen, wird das Annehmen von Geschenken durch Fürsten ganz anders bewertet: Daz liebet eyme fursten tummen ..., Johannes Rothes Ratsgedichte. Nach den Handschriften hg. von Herbert Wolf, Berlin I97 I (Texte des späten Mittelalters und der frühen Neuzeit 25), F III, V. 826.

3I Siehe Schubert/Haase (Anm. I), S. LXXI-LXXIV.

32 Vgl. Lähnemanns (Anm. 20) Fazit S. 190: „Didaktische Verfahrensweisen sind nicht nur ein Weg, jungen Rittern einen Spiegel vorzuhalten, sondern mit ihrer Hilfe können Themen aus allen Lebensbereichen dem Publikum aller Schichten zugänglich gemacht werden." 
